Como citar este artículo: Carvajal-Escobar MD, Gómez-Londoño C, Borja-Gómez W, Sepúlveda-Gallego LE. Fracturas diafisiarias de la clavícula: revisión de la evidencia publicada. Revista Biosalud 2016; 15(1):87-97. DOI: 10.17151/biosa.2016.15.1.10

\title{
FRACTURAS DIAFISIARIAS DE LA CLAVÍCULA: REVISIÓN DE LA EVIDENCIA PUBLICADA
}

\author{
Mario David Carvajal-Escobar ${ }^{1}$ \\ Carlos Gómez-Londoño² \\ Wilmer Borja-Gómez ${ }^{3}$ \\ Luz Elena Sepúlveda-Gallego ${ }^{4}$
}

\section{RESUMEN}

Introducción: La clavícula es uno de los huesos más propensos a lesiones, debido a su localización subcutánea y relativamente anterior; su manejo, ortopédico o quirúrgico, sigue siendo controvertido. Por este motivo, el objetivo que guió la revisión fue justamente identificar las tendencias actuales en cuanto al diagnóstico y al manejo de la fractura de clavícula. Materiales y Métodos: Se realizó una revisión de artículos, usando las bases de datos PubMed/Medline, ScienceDirect, Embase, Ovid, SpringerLink. Se seleccionaron 57 referencias, con base en la calidad de la evidencia presentada por las mismas. Resultados y Discusión: La elección del tratamiento más adecuado para la fractura de clavícula sigue constituyéndose en un reto para el ortopedista. El análisis se debe basar en las características individuales de cada paciente, de una cuidadosa consideración de los beneficios y en los daños relativos de cada intervención, como también en las preferencias del paciente. Para avanzar en la definición de estándares terapéuticos se requiere la realización de estudios que tengan en cuenta un mayor número de individuos especialmente en los extremos de la vida y que permitan generar un mejor nivel de evidencia.
Palabras clave: clavícula, fijación intramedular de fracturas, fracturas del hombro (Fuente: DeCS, BIREME).

\section{DIAPHYSEAL CLAVICLE FRACTURE: REVIEW OF PUBLISHED EVIDENCE}

\section{ABSTRACT}

Introduction: The clavicle is one of the bones more prone to injury because due to their subcutaneous and relative anterior position. Its management, either orthopedic or surgical, remains controversial. Therefore, the objective that guided this review was to identify current trends in the diagnosis and management of broken clavicle. Materials and Methods: a review of articles was conducted using PubMed / Medline, ScienceDirect, Embase, Ovid, and SpringerLink databases. Fifty-seven (57) references were selected based on the quality of the evidence they presented. Results and Discussion: Choosing the most appropriate treatment for broken clavicle continues to be a challenge for the orthopedist. The analysis should be based on the individual characteristics of each patient, a careful consideration of the benefits

\footnotetext{
1 Médico Ortopedista. Profesor Asociado, Departamento Quirúrgico, Universidad de Caldas. Servicio de Ortopedia Hospital de Caldas, Hospital Infantil Universitario. Manizales, Colombia. Correo electrónico: davidcarvajal1@hotmail.com (iD) ORCID: 0000-0001-5982-9460

2 Médico Cirujano. Universidad de Caldas. Servicio de Urgencias, Instituto del Corazón. Manizales, Colombia. carlos.520714266@ucaldas.edu.co iD ORCID: 0000-0003-3844-5964

3 Médico Cirujano. Universidad de Caldas. Unidad de Cuidados Intensivos, Hospital Departamental Santa Sofía. Manizales, Colombia. wilmer2007@hotmail.com (iD ORCID: 0000-0002-1893-4165

4 Ph.D. Profesora Titular, Departamento de Salud Pública, Universidad de Caldas. Manizales, Colombia. lesga@une.net.co iD ORCID: 0000-0002-9220-737X
} 
and the relative harms of each intervention, as well as patient preferences. To advance in the definition of therapeutic standards, are required the performance of studies that take into account a greater number of individuals particularly in the extremes of life, which allow generating a

Las fracturas de clavícula representan un problema epidemiológico relevante que tiene una elevada incidencia en individuos menores de 25 años, activos y saludables, que sufren traumas directos de alta energía en actividades deportivas, caídas desde altura y accidentes de tránsito (1-3).

Habitualmente las fracturas de clavícula se han manejado de forma ortopédica, considerado el manejo quirúrgico solo como una excepción. Incluso, Sócrates postuló que se necesita poco más que una "negligencia benigna" para su manejo. La opción del manejo ortopédico se consolidó en la década de 1960 con los trabajos de C. Neer y C.R. Rowe, quienes reportaron bajas tasas de no consolidación y pseudoartrosis $(0,1 \mathrm{y}$ $0,8 \%$, además de la baja frecuencia de síntomas residuales (4).

Ante este panorama, la comunidad se inclinó hacia el manejo ortopédico, aunque como lo planteó en 2009 Vargas y cols., con la sensación de tener una estadística diferente a la reportada clásicamente y con resultados de pseudoartrosis, mala consolidación y secuelas funcionales superiores a lo reportado $(5,6)$. Los estudios recientes muestran resultados exitosos del manejo quirúrgico y tasas más altas (alrededor de $15 \%$ ) de mala consolidación sintomática y pobres puntajes funcionales, resultantes del tratamiento ortopédico (2-4, 7-9).

Por tal razón, desde el inicio de la década anterior, el tratamiento quirúrgico gradualmente ha ido ganando importancia $\mathrm{y}$, por tanto, se le ha prestado mayor atención a esta lesión, higher level of evidence.

Key words: clavicle, intramedullary fracture fixation, shoulder fractures (Source: MeSH, NLM).

\section{INTRODUCCIÓN}

considerada por algunos autores como "trivial" (10), logrando disminuir las tasas de no consolidación del 15\% al 2,2\% (5).

La fijación externa con placa y tornillos se ha consolidado como el manejo estándar para este tipo de fracturas; sin embargo, la técnica conlleva a una mayor disección de tejidos blandos, riesgo de lesión de nervios supraclaviculares y malos resultados estéticos (11). Dada esta situación, se han realizado intentos por desarrollar técnicas menos invasivas que minimicen estas complicaciones; entre ellas, la fijación intramedular con clavo elástico de titanio ha surgido como una alternativa prometedora, mínimamente invasiva, que permite mantener intacto el periostio, estimular la formación del callo óseo y reducir el tiempo de consolidación (12). En 2013, Edmonds reportó la osteosíntesis con una placa biodegradable absorbible, que está en proceso de evaluación y no requiere retiro del material de osteosíntesis (11).

Actualmente, a pesar de las múltiples opciones disponibles, la elección del tratamiento más adecuado para cada caso sigue siendo un reto para el ortopedista, pues los pacientes -en su mayoría personas jóvenes y activas- esperan una rápida reanudación de las actividades diarias y alivio pronto del dolor, lo que ha llevado a centrarse en el manejo quirúrgico primario para este tipo de fracturas $(13,14)$.

El objetivo de esta revisión es proporcionar un concepto actualizado sobre los aspectos relacionados con la epidemiología, clasificación, diagnóstico y manejo de las fracturas de clavícula. 


\section{Estrategia de búsqueda}

Se realizó la búsqueda usando PubMed/ Medline, ScienceDirect, Embase, Ovid y SpringerLink utilizando las dos primeras fases de la estrategia óptima de búsqueda según el Handbook de Cochrane (http://www.cochrane-handbook.com). Se utilizó el rastreo manual de referencias para completar la búsqueda. Se usaron como descriptores: clavicle, fracture fixationintramedullary, fracture fixation-internal, shoulder fractures (Fuente: MeSH, NLM).

\section{Criterios de inclusión}

Se incluyeron artículos publicados entre 2000 y 2014, de preferencia estudios descriptivos, revisiones sistemáticas, meta-análisis. No se limitó la selección de acuerdo a idioma o edad.

\section{Evaluación de la calidad}

Se usaron dos herramientas para evaluar la calidad metodológica de la selección final de los estudios. Se usó la herramienta de Oxford Center of Evidence Based Medicine (http:/ / www.cebm.net), y la versión modificada de Cochrane Bone, Joint and Muscle Trauma Group (http:/ / www.cochrane-handbook.com).

\section{RESULTADOS}

Se identificaron un total de 1011 referencias, de las cuales 392 fueron potencialmente relevantes después del cribado por título y resumen. El cribado del texto completo resultó en 57 referencias elegibles para la evaluación final de la calidad. No hubo desacuerdo entre los revisores acerca de la selección de los 57 artículos finales.

\section{Anatomía aplicada}

La clavícula es el primer hueso en osificarse hacia el quinto mes de desarrollo fetal $\mathrm{y}$, a diferencia de otros huesos largos, ocurre de forma intramembranosa (2) y su placa de crecimiento medial es la última fisis en cerrarse entre los 22 y 25 años (4).

Es la estructura que transfiere energía del tronco al brazo. Tiene forma de $S$ con una convexidad medial y concavidad lateral. Su extremo acromial es plano, la mitad redonda y su extremo esternal es triangular (2).

En la cara lateral articula con el omoplato y está asegurada por los ligamentos acromioclavicular y coracoclavicular que se insertan en el acromion. En la cara medial la clavícula articula con el esternón y es fuertemente asegurada a la primera costilla, sostenida por el cartílago intraarticular esternoclavicular, los ligamentos costoclaviculares y el músculo subclavio, situación en la que presenta estrecha relación con el plexo braquial, el ápex pulmonar y los vasos subclavios (15).

Es propensa a fracturas debido a su posición subcutánea y a la ausencia de músculos y ligamentos que la protejan de las fuerzas transmitidas constantemente. Cuando se produce la fractura, el músculo esternocleidomastoideo lleva el fragmento medial hacia arriba y atrás, el lateral es llevado hacia abajo por acción de la gravedad y es rotado por acción del músculo pectoral mayor $(2,4)$.

La mala consolidación de la clavícula en asociación con acortamiento ( $\geq 15 \mathrm{~mm}$ ), resulta en escápula alada, cambio en la orientación de la glenoides y angulación hacia arriba de la clavícula y de la articulación esternoclavicular; la cual da como resultado una disminución en el arco de movimiento de los músculos de la cintura escapular (5).

Mecanismo de trauma 
El mecanismo de trauma más frecuente es la caída o golpe directo sobre el hombro (85 a 94\%). Cuando las fuerzas son transmitidas a través del brazo y la mano extendidos, y las fuerzas no son directamente liberadas a la clavícula, es improbable que produzcan fracturas (2 al 5\%) $(2,4)$.

Las fracturas de clavícula en personas jóvenes frecuentemente requieren un mecanismo de alta energía, como aquellas que se presentan por deportes de choque, o colisiones de vehículos de motor. A diferencia de los adolescentes y los adultos jóvenes, la población anciana tiende a fracturarse después de un desplazamiento o caída de baja energía $(2,10,16,17)$.

\section{Epidemiología}

Las fracturas de la clavícula representan entre el 2,6 y el 5\% de todas las fracturas del cuerpo humano $(2,18)$ y constituyen entre el $35 \mathrm{y}$ el $45 \%$ de todas la fracturas de cintura escapular $(10,19,20)$.

En neonatos la incidencia de las fracturas de clavícula varía desde 0,35 a 2,9\% de los nacimientos, y permanecen no detectadas en el momento del alta hospitalaria hasta en un $40 \%$ de los casos (21).

Representan entre el 5 y el 15\% de las fracturas en los niños, siendo la fractura más común en esta edad (22), en los adolescentes representa el $15 \%$ de las fracturas (23) y el 2,6 a $10 \%$ de las fracturas en adultos $(5,24)$. Se ha reportado una incidencia en hombres adultos de 71 por 100.000 , y en mujeres de 30 por 100.000 individuos (1, 2, $20,25)$.

En hombres, el primer pico de incidencia ocurre antes de los 25 años y un segundo pico entre los 55 y los 75 años. En las mujeres la distribución es unimodal, con la incidencia más alta entre la quinta y la sexta década (18). Esta fractura es dos veces más frecuente en hombres (17) y no se han reportado diferencias respecto a las etnias (26).

Las fracturas desplazadas ocurren aproximadamente en el $73 \%$ de todas aquellas que comprometen el tercio medio $(24,27)$ con aparente aumento en la incidencia de conminución, desplazamiento y acortamiento (2). Las fracturas abiertas de la clavícula ocurren en el 0,1 al $1 \%$ de los casos (28).

La tasa de no consolidación es de aproximadamente el $5 \%$ en las fracturas no desplazadas y es mayor en las desplazadas. El acortamiento promedio después de una fractura desplazada es de 1,2 cm. Cuando el rango de acortamiento alcanza 1,4 a $2 \mathrm{~cm}$ representa un punto crítico en el desarrollo de mala consolidación sintomática (26).

En Colombia existe poca información acerca de su epidemiología. En 1990, en el Hospital Universitario del Valle, se encontró una prevalencia de $9,09 \%$ que corresponde a la cuarta fractura más frecuente, presente en personas entre los 18 y 60 años (22).

\section{Sistemas de clasificación}

Existen estudios epidemiológicos a partir de los cuales se han desarrollado varios sistemas de clasificación $(3,4)$ con fines investigativos, meramente descriptivos, de los patrones de fractura de clavícula y no predictivos de resultados. El sistema de clasificación más ampliamente aceptado es el descrito por Allman en 1967 (Tabla 1), en el cual se dividen las fracturas de clavícula según su localización sobre el hueso; sin embargo, brinda poca información acerca de la elección de tratamiento y el posible resultado terapéutico (2).

La Orthopaedic Trauma Association (4) (Tabla $2)$, clasifica las fracturas del tercio medio de forma específica, y ha sido adoptada internacionalmente porque permite, incluso, orientar el tratamiento quirúrgico (2-4). Por su parte, en 1998, Robinson clasifica las 
fracturas del tercio medio de la clavícula (Tabla 3), demostrando niveles satisfactorios de confiabilidad y reproducibilidad intra e interobservador; de igual manera, se requieren estudios para determinar si este sistema puede determinar el tratamiento y los resultados funcionales (2).

Tabla 1. Clasificación Allman de la fractura de clavícula.

\begin{tabular}{lc} 
Tipo & Prevalencia \\
\hline Tipo I (Tercio medio) & $69-82 \%$ - jóvenes \\
\hline Tipo II (Tercio lateral) & $15-25 \%$ - adultos, ancianos \\
\hline Tipo III (Tercio medial) & $5 \%$
\end{tabular}

Fuente: los autores, basados en Jeray (2).

Tabla 2. Clasificación OTA de las fracturas del tercio medio de la clavícula.

\begin{tabular}{lcc} 
Tipo & Descripción & $\begin{array}{c}\text { Tratamiento de } \\
\text { elección }\end{array}$ \\
\cline { 1 - 2 } Tipo A & Lesiones simples con dos fragmentos & Clavo intramedular \\
\cline { 1 - 2 } Tipo B & Lesiones en cuña con un tercer fragmento & \\
\hline Tipo C & Fracturas complejas & Placa
\end{tabular}

Fuente: los autores, basados en Hillen et al. (20).

Tabla 3. Clasificación de Robinson de las fracturas del tercio medio de la clavícula.

Tipo de fractura

\begin{tabular}{lc}
\hline Tipo 2A & 2A1: No desplazada \\
\cline { 2 - 2 } Alineamiento cortical & 2A2: Angulada \\
\hline Tipo 2B & 2B1: Simple o conminuta en cuña \\
Fractura desplazada & 2B2: Aislada o conminuta segmentaria
\end{tabular}

Fuente: los autores, basados en Jeray (2).

\section{Evaluación clínica}

La detección de las fracturas del tercio medio de la clavícula generalmente no es difícil, debido a que durante el interrogatorio y examen físico los pacientes reportan síntomas típicos que suelen ser fácilmente localizados. La ubicación subcutánea de la clavícula permite la apreciación de la tienda de campaña, la palpación de crepitación o deformidad y la equimosis (28). El paciente lesionado usualmente protege y mantiene el brazo afectado cerca del cuerpo.
Además del examen de las funciones vascular, motora y sensitiva, se requiere una adecuada auscultación pulmonar y la valoración de la integridad del plexo braquial, así como la valoración radiológica diagnóstica, en la cual usualmente se deben tomar dos proyecciones: la anteroposterior con el brazo colgando hacia abajo y la proyección con inclinación cefálica a $45^{\circ}$, ya que de este modo se puede clasificar la fractura, determinar el tratamiento y asesorar adecuadamente al paciente $(1,2,4,10)$. La mayoría de los estudios recientes describen 
los resultados usando medidas basadas en el cirujano o radiológicas, para equiparar $(2,4)$. Son pocos los estudios que han reportado los resultados de acuerdo a puntajes basados en el paciente, para lo cual se han validado puntajes como el DASH (Disabilities of the arm, shoulder and hand questionnaire), el Constant, $y$ el Oxford (2), que se pueden revisar en http:/ / www.orthopaedicscore.com/

\section{Opciones de manejo}

La meta primaria del tratamiento de la fractura de clavícula es restaurar la función previa del hombro comprometido. Existe completo acuerdo en la literatura en que la indicación para manejo con reducción cerrada es la presencia de fractura no desplazada y no complicada (9); pero no existe acuerdo general sobre las indicaciones para el manejo con reducción abierta y fijación interna; no obstante, se han planteado algunas como: fractura abierta, desplazamiento severo ( $\geq 21 \mathrm{~mm}$ ), conminución, compromiso neurovascular, no consolidación o retardo, consolidación dolorosa, trauma múltiple, malposición severa y ruptura inminente de la piel, pinzamiento de los tejidos blandos alrededor de la fractura, adolescente mayor y separación persistente de la fractura con una diferencia de más de la mitad del diámetro de la clavícula $(2,19,29)$.

Sehan propuesto como indicaciones relativas para manejo quirúrgico: hombro flotante, inadecuada consolidación dolorosa, acortamiento entre 15-20 mm, fracturas con desplazamiento completo, fracturas conminutas (2), y razones estéticas, las cuales pueden variar entre instituciones $(2,4,18)$. En la población pediátrica el manejo de las fracturas desplazadas sigue siendo controversial; aunque se han obtenido mejores resultados con el enclavamiento intramedular y la osteosíntesis con placa (30).

\section{Tratamiento ortopédico}

La opción de tratamiento óptima para aquellas fracturas no desplazadas consiste comúnmente en inmovilización con cabestrillo o un brace en forma de 8 aplicado en la fase aguda, manteniendo una inmovilización entre dos a seis semanas, de acuerdo con el nivel de confort. En adultos la inmovilización puede extenderse hasta por tres meses. No hay evidencia aún que determine cuál de estas opciones es la mejor (31).

El retorno a los deportes o a la actividad pesada se permite entre las cuatro a seis semanas, después de la consolidación clínica o radiológica. El trabajo ligero con actividad con el brazo por encima del nivel de la cabeza se inicia una vez que el confort lo permita, usualmente de dos a cuatro semanas después de la consolidación (2).

Algunos estudios han mostrado altas tasas de no consolidación sintomática $(51,4 \%)$ y función del hombro reducida por el test de Constant (48,6\%), después del tratamiento no quirúrgico $(32,33)$. Además, para las fracturas manejadas de forma conservadora, es inevitable algún grado de deformidad y acortamiento (34).

\section{Tratamiento quirúrgico}

La osteosíntesis con placa sigue siendo el método estándar para el manejo quirúrgico de las fracturas diafisiarias de clavícula, con altas tasas de éxito y bajas tasas de complicaciones $(11,35)$.

Para este procedimiento se dispone de varios tipos de placas: las placas dinámicas de compresión (DCP), las placas tubulares de tercio caña, las placas de reconstrucción (27) y las placas de clavícula anatómicas, que están moldeadas según la anatomía de la clavícula.

El dispositivo debe ser retirado en más de las dos terceras partes de los casos, debido a la prominencia sintomática. Además, existe el riesgo de daño nervioso con los tornillos de fijación, el cual puede reducirse con un posicionamiento anterior o inferior de la placa 
(26). Este procedimiento implica una gran incisión para posicionar el implante, lo que causa daño tisular adicional (36).

Las complicaciones típicas de este procedimiento son infección, cicatrices hipertróficas, aflojamiento del implante, no consolidación y refractura. Las tasas de infección varían de 0 a $18 \%$ (37), con disminución en los estudios recientes; que reportan dolor e irritación por la presencia del material de osteosíntesis que requiere remoción quirúrgica en el 50 a 100\% de los casos (38). Después de la extracción de la placa, la tasa de refractura está entre el 0 y $8 \%$ y la capsulitis adhesiva del hombro ha sido reportada en el 0 al $7 \%$ de los casos $(2,35)$.

La estabilización intramedular es la técnica ideal desde el punto de vista biomecánico $(11,36)$. Es un procedimiento quirúrgico alternativo para la osteosíntesis con placa, en las fracturas del tercio medio de clavícula, descrito por primera vez por Peroni en 1950 (39).

Se han descrito varias técnicas intramedulares, pero sus resultados funcionales parecen ser inconsistentes $(40,41)$. Los dispositivos usados incluyen los clavos de Hagie, Rockwood, Knowles, Steinmann, Herbert, Kirschner $(15,20,26)$, y la técnica mínimamente invasiva -enclavamiento elástico intramedular- usando un clavo elástico intramedular de titanio (20), técnica descrita por primera vez por Jubel et al. en 2003 (36) y de forma subsiguiente por otros estudios, cada vez con mayor uso $(19,24,35,37$, $38,42,43)$.

La fractura es reducida bajo control de intensificador de imágenes, el clavo es introducido a través del portal medial de entrada del sitio de fractura e impactado lateralmente $(44,45)$. Esta técnica conduce a buenos resultados estéticos y funcionales de forma objetiva y subjetiva. Los pacientes notan la disminución marcada del dolor postoperatorio y una recuperación rápida del rango de movimiento en la articulación del hombro $(4,26)$.

A pesar de que la literatura informa sobre complicaciones significativas usando los clavos Steinmann, Hagie $(41,46)$ y Rockwood $(47)$, los reportes respecto al perfil de complicaciones de los clavos intramedulares usando el clavo elástico intramedular de titanio (TEN - Titanium Elastic Nail), parecen más prometedores $(45,46)$, reflejando una mejoría significativa en la función del hombro y en la reducción del dolor. Además, en la mayor parte de los pacientes, se encuentra una adecuada consolidación biológica y no hay refractura (17).

El clavo elástico intramedular de titanio se ha usado con éxito en la fijación de las fracturas de huesos largos en pediatría, cuya ventaja principal radica en que se puede autobloquear en el hueso y proveer tres puntos de fijación dentro de la clavícula en forma de S (12).

En la Tabla 4 se presentan las ventajas y desventajas de la fijación intramedular respecto a la fijación con placa.

Con esta técnica se han reportado tasas de problemas de consolidación e infecciones profundas, requiriendo la remoción del implante no mayores del 7\%. Sin embargo, las tasas reportadas de complicaciones menores como fallo del implante, rotura, irritación o migración del implante son altas (31\%) (35). La complicación más frecuente es la irritación de la piel, causada por la posición subcutánea del clavo bajo su punto de inserción $(4,7,8,24)$. Estos tipos de implantes no ofrecen estabilidad rotacional y puede ocurrir acortamiento de la clavícula, cuando se aplica en las fracturas conminutas $(35,48)$.

Tabla 4. Ventajas y desventajas del tratamiento 
Mario David Carvajal-Escobar et al.

quirúrgico de la fractura del tercio medio de la clavícula.

\begin{tabular}{|c|c|c|}
\hline & Ventajas & Desventajas \\
\hline Placa & $\begin{array}{c}\text { - Restauración de la longitud de la } \\
\text { clavícula. } \\
\text { - Alineamiento anatómico. } \\
\text { - Mayor compresión y estabilidad } \\
\text { rotacional a través de la línea de fractura. }\end{array}$ & $\begin{array}{c}\text { - Mayor riesgo de rotura o } \\
\text { desprendimiento de la placa. } \\
\text { - Mayor refractura después del retiro } \\
\text { de la placa. } \\
\text { - Mayor estancia hospitalaria (29). }\end{array}$ \\
\hline $\begin{array}{l}\text { Clavo } \\
\text { intramedular }\end{array}$ & $\begin{array}{l}\text { - Menor tamaño de la cicatriz quirúrgica. } \\
\text { - Menor traumatismo de tejidos blandos. } \\
\text { - Mayor estimulación de la formación } \\
\text { copiosa de callo óseo gracias a la } \\
\text { naturaleza de carga compartida. } \\
\text { - Menor estancia hospitalaria. } \\
\text { - Remoción obligatoria del dispositivo (49). }\end{array}$ & $\begin{array}{l}\text { - Prominencia del dispositivo. } \\
\text { - Mayor rotura y migración del } \\
\text { implante. } \\
\text { - Menor estabilidad rotacional. } \\
\text { - Infección del sitio de inserción (50). }\end{array}$ \\
\hline
\end{tabular}

Fuente: los autores.

\section{Comparación de estudios}

Tratamiento quirúrgico versus tratamiento ortopédico: no se encontró diferencia estadísticamente significativa en la mejoría de la función de la extremidad superior a un año de seguimiento. Además, el compromiso nervioso y de la piel, fueron más frecuentes después del manejo quirúrgico. Solo en el estudio de Rehn et al. se encontraron puntajes Constant más altos para el manejo quirúrgico (51). Por el contrario, la rigidez o la limitación de los movimientos del hombro fueron más comunes después del tratamiento conservador $(49,52)$.

Respecto al tratamiento ortopédico, se encontró reducción del $72 \%$ del riesgo relativo para no consolidación, con el uso del clavo intramedular y una reducción del 57\% utilizando placa y tornillos, comparado con el tratamiento ortopédico (49). Se han reportado tasas de no consolidación del 2,2\%, después de la fijación con placa comparada con el 15,1\% después del tratamiento ortopédico $(31,32)$.

Estabilización con placa versus enclavamiento intramedular: en los estudios de Duan et al. y $\mathrm{Xu}$ et al. se encontró un menor grado de dolor con el enclavamiento intramedular a los cuatro días $(53,54)$. Por el contrario, en el estudio de McKee no se hallaron diferencias significativas en la tasa de reintervención, puntajes de función del hombro y tasas de no consolidación e infección, con respecto a estas dos técnicas (55). Con el uso del enclavamiento intramedular se advirtió: menor duración de la cirugía (36 min vs $64 \mathrm{~min})$, estancia hospitalaria promedio $(6,2 \mathrm{vs}$ 9,1 días), y menor longitud delaherida quirúrgica $(4,2 \mathrm{~cm}$ vs $7,8 \mathrm{~cm})(33,54-57)$.

\section{CONCLUSIÓN}

La fractura de la clavícula es un problema de trauma esquelético con una frecuencia muy alta. La elección del tratamiento más adecuado, para cada caso, sigue constituyéndose en un reto para el ortopedista. Las opciones de tratamiento deben ser elegidas con base en las características individuales, después de una cuidadosa consideración de los beneficios y los daños relativos de cada intervención, así como de las preferencias del paciente. Se requieren estudios que tengan en cuenta mayor número de individuos en los extremos de la vida y que permitan generar un mejor nivel de evidencia para la adecuada toma de decisiones clínicas. 


\section{CONFLICTO DE INTERESES}

Los autores declaran no tener ningún conflicto

de intereses.

\section{REFERENCIAS}

1. Pecci M, Kreher JB. Clavicle fractures. Am Fam Physician 2008; 77(1):65-70.

2. Jeray KJ. Acute midshaft clavicular fracture. J Am Acad Orthop Surg 2007; 15(4):239-248.

3. O'Neill BJ, Hirpara KM, O'Briain D, McGarr C, Kaar TK. Clavicle fractures: a comparison of five classification systems and their relationship to treatment outcomes. Int Orthop 2011; 35(6):909-914.

4. Smekal V, Oberladstaetter J, Struve P, Krappinger D. Shaft fractures of the clavicle: current concepts. Arch Orthop Trauma Surg 2009; 129(6):807-815.

5. Alshameeri ZA, Katam K, Alsamaq M, Sonsale P. The outcome of surgical fixation of midshaft clavicle fractures; looking at patient satisfaction and comparing surgical approaches. Int J Shoulder Surg $2012 ; 6(3): 76-81$.

6. Vargas G. Fracturas diafisiarias de la clavícula: comparación entre tratamiento quirúrgico y ortopédico. Rev. Colomb. Ortop. Traumatol. 2009; 23(2):115-119.

7. Eskola A, Vainionpaa S, Myllynen P, Patiala H, Rokkanen P. Outcome of clavicular fracture in 89 patients. Arch Orthop Trauma Surg 1986; 105(6):337-338.

8. Robinson CM, Court-Brown CM, McQueen MM, Wakefield AE. Estimating the risk of nonunion following nonoperative treatment of a clavicular fracture. J Bone Joint Surg Am 2004; 86-A(7):1359-1365.

9. Postacchini R, Gumina S, Farsetti P, Postacchini F. Long-term results of conservative management of midshaft clavicle fracture. Int Orthop 2010; 34(5):731-736.

10. Wijdicks FJ, Houwert RM, Millett PJ, Verleisdonk EJ, Van der Meijden OA. Systematic review of complications after intramedullary fixation for displaced midshaft clavicle fractures. Can J Surg 2013; 56(1):58-64.

11. Edmonds EW. Use of an absorbable plate in the management of a clavicle fracture in an adolescent. Am J Orthop (Belle Mead NJ) 2012; 41(1):29-32.

12. Singh R, Rambani R, Kanakaris N, Giannoudis PV. A 2-year experience, management and outcome of 200 clavicle fractures. Injury 2012; 43(2):159-163.

13. Schiffer G, Faymonville C, Skouras E, Andermahr J, Jubel A. Midclavicular fracture: not just a trivial injury: current treatment options. Dtsch Arztebl Int 2010; 107(41):711-717.

14. Lee YS, Huang HL, Lo TY, Hsieh YF, Huang CR. Surgical treatment of midclavicular fractures: a prospective comparison of Knowles pinning and plate fixation. Int Orthop 2008; 32(4):541-545.

15. Mirzatolooei F. Comparison between operative and nonoperative treatment methods in the management of comminuted fractures of the clavicle. Acta Orthop Traumatol Turc 2011; 45(1):34-40.

16. Namdari S, Ganley TJ, Baldwin K, Rendon Sampson N, Hosalkar H, Nikci V, et al. Fixation of displaced midshaft clavicle fractures in skeletally immature patients. J Pediatr Orthop 2011; 31(5):507-511.

17. Vander Have KL, Perdue AM, Caird MS, Farley FA. Operative versus nonoperative treatment of midshaft clavicle fractures in adolescents. J Pediatr Orthop 2010; 30(4):307-312.

18. Houwert RM, Wijdicks FJ, Steins Bisschop C, Verleisdonk EJ, Kruyt M. Plate fixation versus intramedullary fixation for displaced mid-shaft clavicle fractures: a systematic review. Int Orthop 2012; 36(3):579-585. 
Mario David Carvajal-Escobar et al.

19. Wijdicks FJ, Houwert M, Dijkgraaf M, De Lange D, Oosterhuis K, Clevers G, et al. Complications after plate fixation and elastic stable intramedullary nailing of dislocated midshaft clavicle fractures: a retrospective comparison. Int Orthop 2012; 36(10):2139-2145.

20. Hillen RJ, Burger BJ, Poll RG, De Gast A, Robinson CM. Malunion after midshaft clavicle fractures in adults. Acta Orthop 2010; 81(3):273-279.

21. Malik S, Chiampas G, Leonard H. Emergent evaluation of injuries to the shoulder, clavicle, and humerus. Emerg Med Clin North Am 2010; 28(4):739-763.

22. Concha J, Gerstner J, Gallón L, Bárcenas C. Patrón de fracturas en el Hospital Universitario Cali. Rev. Colomb. Ortop. Traumatol.1990; 4(3):247-250.

23. Stegeman SA, Roeloffs CW, Van den Bremer J, Krijnen P, Schipper IB. The relationship between trauma mechanism, fracture type, and treatment of midshaft clavicular fractures. Eur J Emerg Med 2013; 20(4):268-272.

24. Van der Meijden OA, Gaskill TR, Millett PJ. Treatment of clavicle fractures: current concepts review. J Shoulder Elbow Surg 2012; 21(3):423-429.

25. Bachoura A, Deane AS, Kamineni S. Clavicle anatomy and the applicability of intramedullary midshaft fracture fixation. J Shoulder Elbow Surg 2012; 21(10):1384-1390.

26. Jubel A, Andermahr J. Intramedullary nailing of the clavicle. San Francisco, CA. AAOS march 2008 annual meeting (Abstracts, 75th annual meeting proceedings, 9:302-303).

27. Jubel A, Andermahr J, Schiffer G, Tsironis K, Rehm KE. Elastic stable intramedullary nailing of midclavicular fractures with a titanium nail. Clin Orthop Relat Res 2003; 408:279-285.

28. Arismendi A, Sarassa C. Fractura del tercio lateral de la clavícula en pacientes adolescentes: reporte de caso. Rev. Colomb. Ortop. Traumatol 2009; 23(4):229-233.

29. Zlowodzki M, Zelle BA, Cole PA, Jeray K, McKee MD. Treatment of acute midshaft clavicle fractures: systematic review of 2144 fractures: on behalf of the Evidence-Based Orthopaedic Trauma Working Group. J Orthop Trauma 2005; 19(7):504-507.

30. Caird MS. Clavicle shaft fractures: are children little adults? J Pediatr Orthop 2012; 32(Suppl 1):S14.

31. Bajuri MY, Maidin S, Rauf A, Baharuddin M, Harjeet S. Functional outcomes of conservatively treated clavicle fractures. Clinics (Sao Paulo) 2011; 66(4):635-639.

32. Shin SJ, Do NH, Jang KY. Risk factors for postoperative complications of displaced clavicular midshaft fractures. J Trauma Acute Care Surg 2012; 72(4):1046-1050.

33. Simon P. Mid-clavicle fractures in adults: surgery or not? Eur J Orthop Surg Traumatol 2010; 20(7):521525.

34. Liu GD, Tong SL, Ou S, Zhou LS, Fei J, Nan GX, Gu JW. Operative versus non-operative treatment for clavicle fracture: a meta-analysis. Int Orthop 2013; 37(8):1495-1500.

35. Wijdicks FJ, Van der Meijden OA, Millett PJ, Verleisdonk EJ, Houwert RM. Systematic review of the complications of plate fixation of clavicle fractures. Arch Orthop Trauma Surg 2012; 132(5):617-625.

36. Fu TH, Tan BL, Liu HC, Wang JW. Anatomical reduction for treatment of displaced midshaft clavicular fractures: Knowles pinning vs. reconstruction plating. Orthopedics 2012; 35(1):e23-30.

37. Paul SP, Heaton PA, Patel K. Breaking it to them gently: fractured clavicle in the newborn. Pract Midwife 2013; 16(9):31-34.

38. Kleweno CP, Jawa A, Wells JH, O’Brien TG, Higgins LD, Harris MB, et al. Midshaft clavicular fractures: comparison of intramedullary pin and plate fixation. J Shoulder Elbow Surg 2011; 20(7):1114-1117.

39. Peroni L. Medullary osteosynthesis in the treatment of clavicle fractures. Arch Ortop 1950; 63(4):398405.

40. Assobhi JE. Reconstruction plate versus minimal invasive retrograde titanium elastic nail fixation for displaced midclavicular fractures. J Orthop Traumatol 2011; 12(4):185-192. 
41. Walz M, Kolbow B, Auerbach F. Elastic, stable intramedullary nailing in midclavicular fractures - a change in treatment strategies? Unfallchirurg 2006; 109(3):200-211.

42. Lee HJ, Oh CW, Oh JK, Yoon JP, Kim JW, Na SB, Kyung HS. Percutaneous plating for comminuted midshaft fractures of the clavicle: a surgical technique to aid the reduction with nail assistance. Injury 2013; 44(4):465-470.

43. Millett PJ, Hurst JM, Horan MP, Hawkins RJ. Complications of clavicle fractures treated with intramedullary fixation. J Shoulder Elbow Surg 2011; 20(1):86-91.

44. Lenza M, Belloti JC, Andriolo RB, Gomes Dos Santos JB, Faloppa F. Conservative interventions for treating middle third clavicle fractures in adolescents and adults. Cochrane Database Syst Rev 2009; 15(2): CD007121.

45. McKee RC, Whelan DB, Schemitsch EH, McKee MD. Operative versus nonoperative care of displaced midshaft clavicular fractures: a meta-analysis of randomized clinical trials. J Bone Joint Surg Am 2012; 94(8):675-684.

46. Stegeman SA, et al. Displaced midshaft fractures of the clavicle: non-operative treatment versus plate fixation (Sleutel-TRIAL). A multicentre randomised controlled trial. BMC Musculoskelet Disord $2011 ; 12: 196$.

47. Mudd CD, Quigley KJ, Gross LB. Excessive complications of open intramedullary nailing of midshaft clavicle fractures with the Rockwood Clavicle Pin. Clin Orthop Relat Res 2011; 469(12):3364-3370.

48. Liu PC, Chien SH, Chen JC, Hsieh CH, Chou PH, Lu CC. Minimally invasive fixation of displaced midclavicular fractures with titanium elastic nails. J Orthop Trauma 2010; 24(4):217-223.

49. Kulshrestha $V$, Roy $T$, Audige L. Operative versus nonoperative management of displaced midshaft clavicle fractures: a prospective cohort study. J Orthop Trauma 2011; 25(1):31-38.

50. Martetschläger F, Gaskill TR, Millett PJ. Management of clavicle nonunion and malunion. J Shoulder Elbow Surg 2013; 22(6):862-868.

51. Rehn $\mathrm{CH}$, Kirkegaard M, Viberg B, Larsen MS. Operative versus nonoperative treatment of displaced midshaft clavicle fractures in adults: a systematic review. Eur J Ortopedic Surg Traumatol 2013. Available from: 10.1007/s00590-013-1370-3

52. Lenza M, Buchbinder R, Johnston RV, Belloti JC, Faloppa F. Surgical versus conservative interventions for treating fractures of the middle third of the clavicle. Cochrane Database Syst Rev 2013; 6:CD009363.

53. Duan X, Zhong G, Cen S, Huang F, Xiang Z. Plating versus intramedullary pin or conservative treatment for midshaft fracture of clavicle: a meta-analysis of randomized controlled trials. J Shoulder Elbow Surg 2011; 20(6):1008-1115.

54. Xu CP, Li X, Cui Z, Diao XC, Yu B. Should displaced midshaft clavicular fractures be treated surgically? A meta-analysis based on current evidence. Eur J Orthop Surg Traumatol 2013; 23(6):621-629.

55. McKee MD. Clavicle fractures in 2010: sling/swathe or open reduction and internal fixation? Orthop Clin North Am 2010; 41(2):225-231.

56. Lenza M, Belloti JC, Gomes Dos Santos JB, Matsumoto MH, Faloppa F. Surgical interventions for treating acute fractures or non-union of the middle third of the clavicle. Cochrane Database Syst Rev 2009; 4:CD007428.

57. Kadakia AP, Rambani R, Qamar F, McCoy S, Koch L, Venkateswaran B. Titanium elastic stable intramedullary nailing of displaced midshaft clavicle fractures: A review of 38 cases. Int J Shoulder Surg 2012; 6(3):82-85. 